\title{
Neuro-ophthalmic pathological correlations in primary reticulum cell sarcoma of the nervous system
}

\author{
E. F. GONYEA ${ }^{1}$ AND G. H. COLLINS ${ }^{2}$ \\ From the Veterans Administration Hospital and Divisions of Neurology and Neuropathology, \\ University of Florida College of Medicine, Gainesville, Florida 32602, U.S.A.
}

SYNOPSIS Two cases of primary reticulum cell sarcoma of the nervous system are presented because of their unusual clinicopathological correlations. Consecutive pupil-sparing third nerve paralysis was associated with tumour infiltrating the diencephalon and mesencephalon and the fascicular portions of both oculomotor nerves in the first case. Concomitant sympathetic involvement, as evidenced by relative miosis, cannot account for the preserved pupillary reactions. Preservation of the pupils was most likely the result of the intrinsic rather than compressive, third nerve involvement. In the second case involvement of the right facial colliculus was associated with the 'one-and-a-half' syndrome (combined lateral gaze palsy and unilateral internuclear ophthalmoplegia) and peripheral facial nerve paralysis. Terminally, the left lateral rectus muscle was also involved, and a discrete tumour implant had extended well beyond the midline.

Pupil-sparing third nerve paralysis immediately arouses suspicion of diabetic, hypertensive, or arteritic involvement of the oculomotor nerve (Smith, 1970). The 'one-and-a-half syndrome' (combined lateral gaze palsy and unilateral internuclear ophthalmoplegia) is most commonly seen with vascular disease of the brain-stem and only rarely with demyelinative or neoplastic lesions (Fisher, 1967). Two patients, each with one of these clinical presentations due to primary reticulum cell sarcoma of the nervous system (RCS), are the subject of this report.

\section{CASE 1}

A 64 year old white man had onset of double vision three months before death. He had been well until he complained of headaches and his wife noted personality change over a two or three week interval. When hospitalized five days after the onset of diplopia, examination revealed a right oculomotor nerve paralysis sparing the pupil, and a left extensor plantar response. Because of increasing lethargy during the second hospital week he was transferred to this hospital.

1 Present address: Neurology Service, Veterans Administration Hospital, Memphis, TN38104, U.S.A.

2 Present address: State University of New York, Upstate Medical Center, Syracuse, N.Y., U.S.A.
There was a xanthelasma of the left lower eyelid, otherwise the general examination was unremarkable. He was lethargic, though readily arousable. He was confused and disoriented but had no specific deficit of higher cortical function. There was a right exotropia and oculomotor paralysis with ptosis and external ophthalmoplegia. Abduction and intorsion on attempted depression were preserved. The left eye was uninvolved. The pupils were $2.5 \mathrm{~mm}$ diameter and reacted to light through a small arc. There was slight left central facial weakness and a slight left hemiparesis with an extensor plantar response. The fundi appeared normal.

In the ensuing week he became more alert and the left hemiparesis became more obvious. Three weeks after the initial right oculomotor nerve involvement, left oculomotor nerve paralysis, again sparing the pupil, evolved over a 48 hour period. The eyes were divergent with bilateral ptosis and external ophthalmoplegia. Abduction and intorsion on attempted aepression were bilaterally preserved. There was definite monocular abducting nystagmus in either eye on following movements. Ice water caloric tests produced the anticipated dissociated nystagmus. Neither adducting eye moved beyond the midline. Convergence was absent. The pupils were spared and no ciliospinal reflex was elicited. They became dilated and fixed for 36 hours with mydriatics.

In the next two months he became progressively obtunded and developed bilateral extensor plantar 

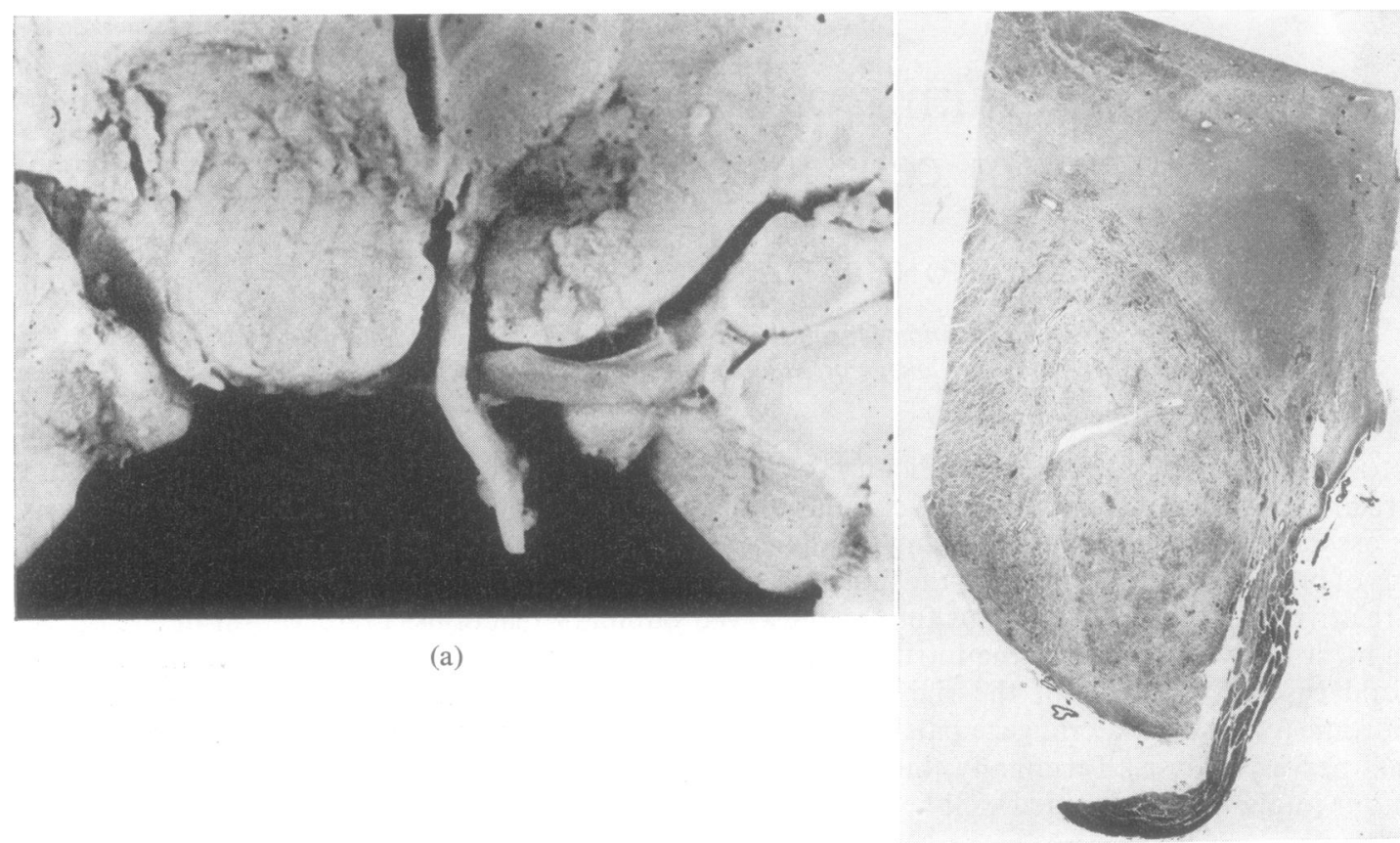

(a)

(b)

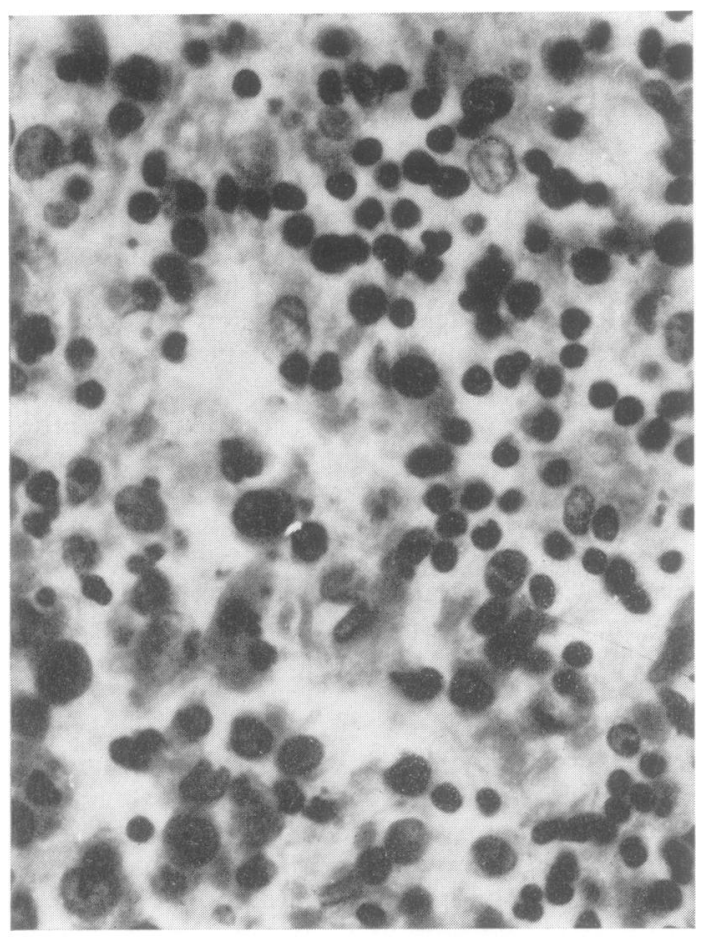

FIG. 1. Case 1. (a, top left) Tumour involving the basis pedunculi and extending into the zone of exit of $\frac{\mathrm{O}}{\mathrm{D}}$ the left oculomotor nerve. (b, top right) $A$ myelinstained section showing tumour in the right cerebral $\overrightarrow{\overrightarrow{0}}$ peduncle extending into the root of the third nerve. (c, left) This photomicrograph of the mesencephalon is illustrative of the cell types in this tumour. $H$ and $E$, $\times 320$.

(c) 
responses. The course was complicated by intermittent low grade fevers and pneumonia. A feeding gastrostomy was performed. He developed a urinary tract infection which was treated appropriately. He continued to do poorly and terminally developed fever to $42 \cdot 2^{\circ} \mathrm{C}$ and dilated, fixed pupils in association with respiratory arrest.

Laboratory examinations revealed abnormal glucose tolerance and hyperlipidaemia. Skull and chest radiographs were unremarkable. Plasma thyroxine $\left(\mathrm{T}_{4}\right)$ was $5.5 \mu \mathrm{g} / 100 \mathrm{ml}$. Ten milligrams of edrophonium chloride (Tensilon) intravenously afforded no response. LE preparations were negative. Erythrocyte sedimentation rate was $31 \mathrm{~mm}$ in the first hour. Serum protein electrophoresis revealed slight hyper-alpha-2 globulinaemia. Serological tests for syphilis on serum and cerebrospinal fluid (CSF) were non-reactive. Three lumbar punctures were performed throughout his illness. All revealed clear CSF under normal pressure with no white cells and protein contents ranging from 98 to $128 \mathrm{mg} / 100 \mathrm{ml}$.

An electroencephalogram (EEG) revealed diffuse low voltage theta slowing. Technetium brain scans at the previous hospital and on this admission revealed an increased area of uptake superior and posterior to the sellar region. A right carotid angiogram on admission and a right brachial angiogram after bilateral third nerve involvement were normal.

NECROPSY The general pathological examination was unremarkable.

On gross examination the brain demonstrated a massive distortion involving the left cerebral peduncle particularly with a shift of midline structures to the right side. Cut sections revealed a greyish granular and somewhat friable mass which obliterated normal structures and extended from the level of the anterior commissure to the rostral pons, bilaterally (Fig. 1a). In this region the tumour involved the inferior and medial portion of the thalamus, the hypothalamus, the subthalamus, the majority of the mesencephalic tegmentum and basis pedunculi (Fig. 1b) terminating in the basis pontis. The tumour extended laterally into the inferior portion of the internal capsule and terminated in the globus pallidus. The lateral ventricles were moderately dilated but no other abnormalities were seen. On microscopic examination (Fig. 1c) the tumour was highly cellular and invading the adjacent brain tissue in which a reactive gliosis was seen. Perivascular growth of small mononuclear cells was noted, particularly in these junctional areas. The main tumour mass consisted of two principal cell types. Small round mononuclear cells with relatively scant cytoplasm occurred sometimes in clusters but were usually spread diffusely among the other tumour cells. The second type was characterized by large hyperchromatic nuclei which were multilobed and occasionally contained round eosinophilic inclusion material. The cytoplasm of these cells was frequently voluminous and contained basophilic particulate material. The Wilder stain for reticulin fibres demonstrated an intrinsic network of thin delicate fibres in many, though not all, areas of the tumour. A diagnosis of reticulum cell sarcoma was made.

\section{CASE 2}

A 60 year old black man was in good health until eight months before death. In the ensuing five months he lost $13.6 \mathrm{~kg}$ in weight and complained of right-sided head pain. He noted fluttering of his right eyelids and later swelling and redness of the eye for which he sought medical attention and right facial weakness was noted. This progressed until he was unable to close the right eye. Thereafter he experienced a one hour episode of loss of vision in the right eye followed by double vision. Subsequently the only horizontal movement remaining in either eye was outward movement of the left eye. This visual disturbance was associated with some unsteadiness in walking and he also experienced some numbness and weakness of his right arm that resolved after a month.

When admitted to this hospital three months before death, he was afebrile and the general physical examination was unremarkable. No abnormality was found in the nasopharynx. Neurological examination revealed mild ataxia of tandem gait, hyperactive muscle stretch reflexes without pathological responses and severe right peripheral facial paralysis. Neuro-ophthalmological examination revealed right horizontal conjugate gaze paralysis and right medial rectus paralysis associated with abducting nystagmus in the left eye. Convergence, vertical movements of both eyes, and abduction of the left eye were preserved. There was some mild vertical upbeating nystagmus. Visual acuity and fields, pupils, and fundi were normal. The right palpebral fissure was widened and he was unable to close the right eye. In the month before death he developed mild anisocoria with a smaller left pupil and limitation of left lateral rectus function.

Laboratory examinations revealed a mild leucocytosis of 10 to 16,000 cells per $\mathrm{mm}^{3}$ and anaemia with a packed cell volume of $30 \%$. Serological tests for syphilis on serum and CSF were non-reactive. Skull and chest radiographs were unremarkable. An EEG was normal. Two technetium brain scans showed an increased area of uptake anterior and superior to the sellar region. Repeated lumbar and a 

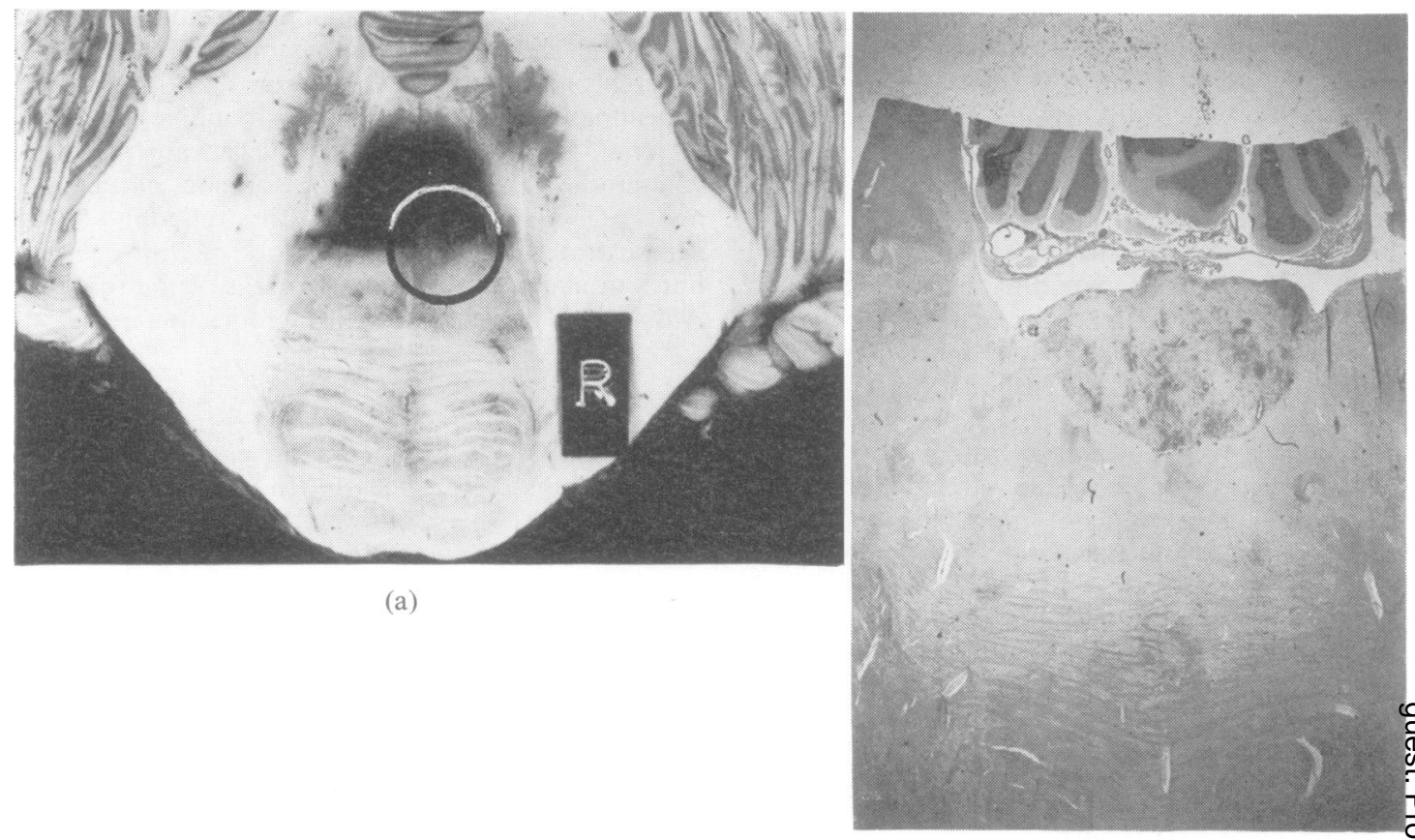

(a)

(b)

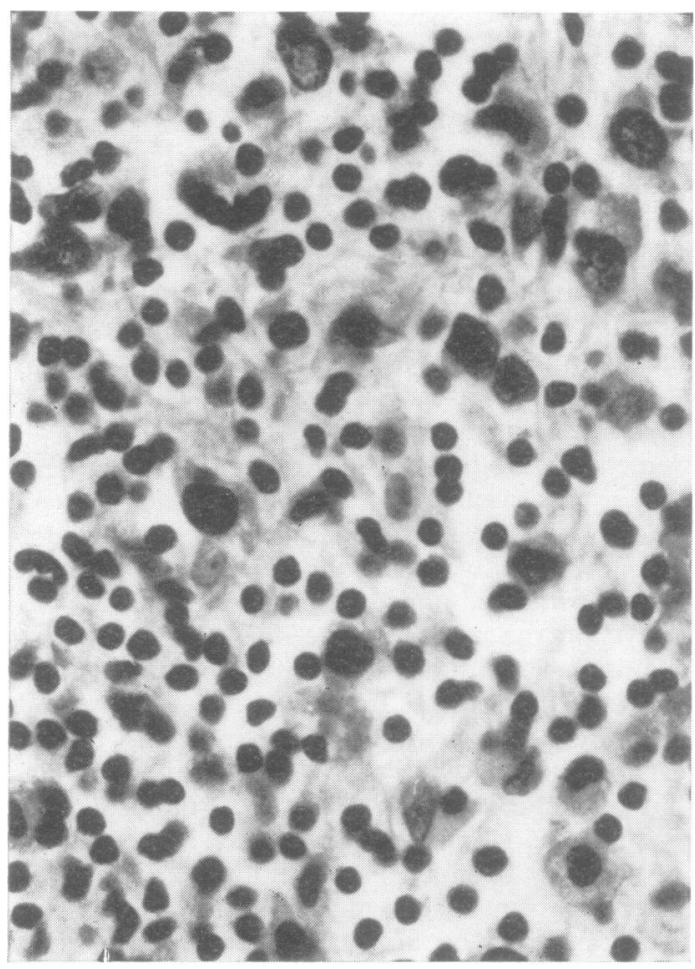

FIG. 2. Case 2. (a, top left) The caudal pons demonstrates the tumour nodule involving the region of the right facial colliculus, though clearly extending beyond the midline. (b, top right) Tissue section of the pons showing tumour implant in the floor of the fourth ventricle involving both facial colliculi. $L F B-H$ and $E$. (c, left) The histological features of this tumour are similar to those of case $1 . H$ and $E, \times 320$.

(c) 
cisternal puncture revealed clear CSF under normal pressure with 77 to 128 white cells per $\mathrm{mm}^{3}(37-100 \%$ lymphocytes), $109-144 \mathrm{mg}$ per $100 \mathrm{ml}$ protein, and 47-85 mg per $100 \mathrm{ml}$ sugar. Tumour cells were subsequently identified on two occasions, once from the lumbar route and again from ventricular fluid at the time of ventriculography. There was no growth on extensive culturing of CSF, urine, liver, and bone marrow. Selective catheterization and angiography of both common carotid arteries and the left vertebral artery were normal. Two pneumoencephalograms and a ventriculogram cumulatively confirmed the presence of a midline mass indenting the anterior horns of both lateral ventricles more so on the right.

The patient's course was stable. A right lateral tarsorrhaphy was performed. Two weeks before death, biopsy of a ventricular mass showed undifferentiated malignant tumour with inflammation. Postoperatively the patient did well the first day but then became unresponsive, requiring intubation and intravenous steroids. He responded dramatically and was walking by the sixth postoperative day. On the ninth day he developed an acute thrombophlebitis of the deep veins of the right leg, was put back to bed and treated with anticoagulants, being maintained with a prothrombin time $2 \frac{1}{2}$ times normal. Six days later he suddenly became unresponsive. Anticoagulants were discontinued but he died eight hours later.

NECROPSY There were bilateral pulmonary emboli with an organized infarct in the left lung.

The brain had a surgical defect in the right frontal lobe, surrounding which the brain tissue was swollen and tense and shifted to the left of the midline. Sections of the brain demonstrated a nodular and highly friable tumour which extended from the septal region superiorly into the rostrum of the corpus callosum and posteriorly along the head and body of the right caudate nucleus to the level of the mammillary bodies. The anterior aspect of this mass was involved with haemorrhagic changes thought to represent the site of previous surgery. Coextensive with the anterior portion of the tumour was a projection of granular and friable tissue into the floor of the right lateral ventricle adjacent to the foramen of Monro. There were several other ependymal nodules studding the frontal horns of both lateral ventricles. One other nodule was noted on the ependymal surface of the fourth ventricle at the level of the facial colliculus which extended into the parenchyma a distance of 3 to $5 \mathrm{~mm}$ (Fig. 2a, b). Microscopic examination of the areas of tumour demonstrated a highly invasive cellular tumour with frequent mitoses and a perivascular growth pattern at the margins. Tumour cells were of two fairly distinct types with intermediate forms. Many small mononuclear cells with scanty cytoplasm were intermingled with much larger cells consisting of hyperchromatic pleomorphic nuclei and voluminous cytoplasm (Fig. 2c). Mitotic figures were frequently seen. The reticulin stain demonstrated a network of thin delicate silver-positive fibres. Reticulum cell sarcoma was diagnosed.

\section{DISCUSSION}

The pathological condition described in both of these patients is typical of primary lymphoreticular malignancy of brain. Despite the several names which have been applied to this tumour in the past, it is now recommended by some (Vuia and Mehraein, 1971; Schaumburg et al., 1972) that the compound term of reticulum cell sarcoma-microglioma be employed. We do not intend to address the controversy which has been associated with this tumour but rather to establish that the pathology of these two cases is representative.

Histologically, the tumours were very similar. Both were highly invasive of brain tissue and demonstrated perivascular growth. Both also contained small mononuclear cells and large cells with multilobuled and hyperchromatic nuclei many of which were certainly phagocytic. As has been described in the more undifferentiated forms (Vuia and Mehraein, 1971; Schaumburg et al., 1972; Rubinstein, 1972), the reticulin pattern was poorly developed. In case 2 , mitotic figures were, however, much more frequent.

The occurrence of this tumour as a primary neoplasm of brain may, in fact, be the most frequent manner of presentation (Schaumburg et al., 1972). The growth pattern and distribution in each of these cases are, in their own way, characteristic of this tumour. In case 1 , the complete involvement of the mesencephalicdiencephalic core of brain tissue is a well recognized pattern. In case 2 , the tumour presented a deep frontal lobe mass which was periventricular in distribution with several remote tumour deposits on the ventricular surface. The ocular manifestations occurring in these two patients were, quite obviously, therefore, the result of two rather distinct patterns of growth of the same tumour type.

Lesions of the oculomotor nerves most commonly produce both an external (with preserva- 
tion of abduction and intorsion on attempted depression) and internal ophthalmoplegia. Exceptions in which the pupil alone is involved or spared are well known (Walsh and Hoyt, 1969). The former occurs with compression in tentorial herniation and the latter with ischaemic infarction. In either case, the peripheral or superficial distribution of the pupillomotor fibres (Sunderland and Hughes, 1946) has been held responsible. It is obvious that the pupillary involvement may be incomplete or the sparing relative.

Concomitant involvement of sympathetic nerve fibres might account for relative preservation of pupillary size, but could not account for preserved pupillary reactions (Cogan, 1956). Combined involvement of afferent fibres to oculomotor nuclei in the midbrain tectum, sympathetics, and oculomotor nerve paralysis with preservation of pupillomotor fibres (as might theoretically occur, for example, with syphilis) would be associated with loss of pupillary reactions to light.

While sympathetic involvement is likely in our first case because of the miosis and obvious hypothalamic involvement by the tumour, there can be little doubt that the parasympathetic pupillomotor fibres were relatively, if not completely, spared by the process. The clinical course in this patient was that of a progressive infiltrating tumour without an associated increase in intracranial pressure.

Tumour was found to be infiltrating throughout the diencephalon and mesencephalon and had penetrated and infiltrated both oculomotor nerves. While we cannot state with certainty why the pupillomotor fibres were preserved, we would like to suggest that this was the result of intrinsic rather than compressive involvement of the oculomotor nerves. In this regard, involvement was analogous to the previously reported and pathologically documented cases of diabetic oculomotor nerve paralysis (Dreyfus et al., 1957; Asbury et al., 1970; Weber et al., 1970).

The monocular abducting nystagmus observed in this patient is of at least passing interest. While tumour did not grossly involve the dorsal tegmentum, we cannot exclude concomitant microscopic involvement of the medial longitudinal fasciculus (MLF).

The facial colliculi are paramedian eminences projecting into the floor of the fourth ventricle at the level of the abducens nuclei in the pons. This eminence is formed by fibres of the facial nerve as they pass around the abducens nucleus. Related structures include the MLF and paramedian pontine reticular formation.

Sixth and seventh nerve paralysis, internuclear ophthalmoplegia, and lateral gaze paralysis in varying combinations are anticipated with? lesions in this area. Extension of such a lesion $\underset{\overrightarrow{\mathrm{F}}}{\overrightarrow{\mathrm{S}}}$ across the midline would be indicated by contralateral lateral rectus muscle or facial nerve dysfunction.

The 'one-and-a-half' syndrome-that is, com- $\stackrel{\Phi}{\circ}$ bined ipsilateral gaze palsy and internuclear ophthalmoplegia - does not necessarily indicate involvement at the level of the facial colliculus (abducens nucleus). Inasmuch as the supranuclear pathways for lateral gaze decussate at or about the level of the trochlear nucleus (Daroff and Hoyt, 1971), this syndrome may be see with lesions at any level between these nuclei. $\infty_{0}^{\circ} 0$

With the 'one-and-a-half' syndrome there if $\overrightarrow{0}$ clinical difficulty in assessing ipsilateral abducen 80 and contralateral MLF function. Involvement of 9 the former can be established with certainty onl if there is an exotropia. There are no firm clinicat criteria to distinguish the latter, though certainlg the MLF would be the first structure to be in 3 volved with extension across the midline at the level. Convergence would be expected to be pre $\overrightarrow{0}$ served whether or not the contralateral MLF were involved. We have considered that an exotropia might indicate contralateral involvement, but certainly this is subject to varying interpretation. When he coined the term 'oneand-a-half' syndrome, Fisher (1967) described exotropia as an inherent feature. Daroff and Hoyt (1971) regard exotropia in internuclear ophthalmoplegia as indicative of involvement of the oculomotor nucleus. Cogan (1970), on the other hand, has difficulty reconciling why the majority of patients with internuclear ophthalmoplegia are orthotropic in the primary position of gaze. It is pertinent to this discussion that our second case had no tropia and had both ipsilateral abducens and contralateral MLF involvement.

Although clinicopathological correlations have been made with lesions of the fascicular portion of the sixth nerve in the dorsolateral (Foville's syndrome) and ventromedian (Millard-Gubler 
syndrome) portions of the pons (Walsh and Hoyt, 1969) and to some extent in the 'one-and-a-half' syndrome (Castleman, 1953; Enoksson, 1956; Cogan and Wray, 1970), we believe this is the first clinicopathological correlation that has been made in what we would designate as the syndrome of the facial colliculus.

Pathologically there is no question of bilateral involvement, the lesion is maximal on the right, but the clinical course would indicate that extension across the midline was a late occurrence.

Schaumburg et al. (1972) recently reviewed their experience with primary reticulum cell sarcoma. In a few of their patients neuroophthalmological features were prominent. Our two patients, while not diagnosed during life, conformed well with their first pattern of neurological symptomatology that should have raised the suspicion of primary reticulum cell sarcoma: 'a meningo-cerebral lesion with focal neurological signs and signs of meningeal inflammation without an infective agent being found. The course is of a steady increase of a neurological deficit, usually a succession of cranial-nerve palsies, nuchal rigidity and stupor.' The importance of the antemortem diagnosis lies in their favourable experience with radiotherapy.

\section{REFERENCES}

Asbury, A. K., Aldredge, H., Hershberg, R., and Fisher, C. M. (1970). Oculomotor palsy in diabetes mellitus: a clinico-pathological study. Brain, 93, 555-566.

Castleman, B. (ed.) (1953). Case records of the Massachusetts General Hospital, case 39451. New England Journal of Medicine, 249, 776-780.
Cogan, D. G. (1956). Neurology of the Ocular Muscles. Second edn, pp. 61-68. Thomas: Springfield, Ill.

Cogan, D. G. (1970). Internuclear ophthalmoplegia, typical and atypical. Archives of Ophthalmology, 84, 583-589.

Cogan, D. G., and Wray, S. H. (1970). Internuclear ophthalmoplegia as an early sign of brainstem tumors. Neurology (Minneap.), 20, 629-633.

Daroff, R. B., and Hoyt, W. F. (1971). Supranuclear disorders of ocular control systems in man. In The Control of Eye Movements, pp. 175-235. Edited by P. Bach-y-Rita and C. C. Collins. Academic Press: New York.

Dreyfus, P. M., Hakim, S., and Adams, R. D. (1957). Diabetic ophthalmoplegia. Archives of Neurology and Psychiatry (Chic.), 77, 337-349.

Enoksson, P. (1965). Internuclear ophthalmoplegia and paralysis of horizontal gaze. Acta Ophthalmologica, 43, 697-707.

Fisher, C. M. (1967). Some neuro-ophthalmological observations. Journal of Neurology, Neurosurgery, and Psychiatry, 30, 383-392.

Rubinstein, L. J. (ed.) (1972). Tumors of the lymphoreticular system. In Atlas of Tumor Pathology, 2nd series, fasc. 6, pp. 215-234. Armed Forces Institute of Pathology: Washington.

Schaumburg, H. H., Plank, C. R., and Adams, R. D. (1972). The reticulum cell sarcoma-microglioma group of brain tumours. Brain, 95, 199-212.

Smith, J. L. (ed.) (1970). Neuro-ophthalmology. Volume 5. pp. xii. Huffman: Hallandale, Florida.

Sunderland, S., and Hughes, E. S. R. (1946). The pupilloconstrictor pathway and the nerves to the ocular muscles in man. Brain, 69, 301-309.

Vuia, O., and Mehraein, P. (1971). Primary reticulosis of the central nervous system. Journal of Neurological Sciences, 14, 469-482.

Walsh, F. B., and Hoyt, W. F. (1969). Clinical Neuroophthalmology. Third edn, 3 vols, pp. 247-248 (vol. 1), 1110 (vol. 2), 2403 (vol. 3). Williams and Wilkins: Baltimore.

Weber, R. B., Daroff, R. B., and Mackey, E. A. (1970). Pathology of oculomotor nerve palsy in diabetics. Neurology (Minneap.), 20, 835-838. 